\title{
Wave scattering from self-affine surfaces
}

\author{
Ingve Simonsen*†, Damien Vandembroucq* and Stéphane Roux* \\ *Laboratoire CNRS/Saint-Gobain "Surface du Verre et Interfaces", 93303 Aubervilliers Cedex, France \\ ${ }^{\dagger}$ Department of Physics, The Norwegian University of Science and Technology, N-7491 Trondheim, Norway
}

(September 10, 2018)

\begin{abstract}
Electromagnetic wave scattering from a perfectly reflecting self-affine surface is considered. Within the framework of the Kirchhoff approximation, we show that the scattering cross section can be exactly written as a function of the scattering angle via a centered symmetric Lévy distribution for general roughness amplitude, Hurst exponent and wavelength of the incident wave. The amplitude of the specular peak, its width and its position are discussed as well as the power law decrease (with scattering angle) of the scattering cross section.
\end{abstract}

42.25.Fx, 68.35.Ct, 05.45.Df

Wave scattering from rough surfaces has been studied for long [1,2] (see also [3 5] for recent reviews of the subject) with potential applications in remote sensing, acoustical/optical/radar detection or design of surfaces with specified scattering properties... The theoretical prediction of the angular distribution of the scattered intensity requires a proper statistical description of the surface roughness. At this stage it is classical to assume that the statistics of the height and its correlation function are Gaussian. This assumption allows to build analytical expressions and to compare them with experimental or numerical results.

Since the pioneering work of Mandelbrot [6], however, scale invariance has emerged as a relevant tool to describe the geometry of real "disordered" objects. In the case of surfaces, the scale invariance takes the form of self-affinity. Such surfaces remain invariant under dilation of different ratios over the horizontal and the vertical directions. This long range correlated height distribution is characterized by a roughness exponent, an amplitude parameter, and by the lower and upper limits of the scaling invariance region (instead of a height standard deviation and a two point correlation function of finite width). It turned out that many real surfaces can be described through this formalism. Surfaces obtained from fracture [7 [9], growth or deposition processes [10] are classical examples. Similar results have been more recently found for surfaces obtained from cold metal rolling 111. Note that such scaling invariance properties are exhibited from nanometric scales for native float glass 12 up to kilometric scales for geological faults [13].

The aim of this letter is to exhibit an analytical expression of the distribution of the scattering cross section for a perfectly reflecting self-affine surface in the framework of the Kirchhoff approximation. Since a first paper examining the effect of scale invariance in the context of scattering from rough surfaces [14], a large amount of studies have been published in various journals (see for example Ref. [15 23]). Two main points motivated most of these studies $i$ ) the effect of scale invariance on the field scattered from the surface in comparison with the more classical case of a finite width correlation length ii) the hope of directly measuring the "fractal" parameters of the surface via an acoustical or optical experiment. In spite of a large amount of works, it is fair to say that concerning far field scattering the only stable result that emerged is the existence of a power law tail in the intensity distribution with an exponent directly related to the roughness exponent. Very few analytical predictions can be found and oppositely a large number of numerical simulations still lack a clear physical interpretation.

A reason for this could be an excessive focus on the "fractal dimension" (conversely the roughness exponent) with a blind point on the other parameters (the limits of the scale invariance regime and more often the amplitude parameter). A noteworthy exception is, however, due to Jakeman and his collaborators 24,25] who predicted a Lévy distribution for the intensity of a wave scattered from a self-affine random phase screen. However, these authors did not extend these analytical results to the case of scattering from surfaces.

In the following we briefly recall the main properties of self-affine surfaces and we use the Kirchhoff approximation to show that the scattering cross section can be written as a function of the scattering angle via a centered symmetric Lévy distribution.

A surface is self-affine between the scales $\xi_{-}$and $\xi_{+}$ if it remains (either exactly or statistically) invariant in this region under transformations of the form:

$$
(x, y, z) \rightarrow\left(\lambda x, \lambda y, \lambda^{H} z\right) .
$$

The exponent, $H$, is usually called the roughness or Hurst exponent. Restricting ourselves to profiles, a statistical translation of the previous statement is that the probability $p(\Delta z ; \Delta x)$ of having a height difference $\Delta z$ over the distance $\Delta x$ or its cumulative $\mathcal{P}(\Delta z ; \Delta x)=$ $\int_{-\infty}^{\Delta z} p(\delta z ; \Delta x) d \delta z$ is such that:

$$
\mathcal{P}(\Delta z ; \Delta x)=\mathcal{P}\left(\lambda^{H} \Delta z ; \lambda \Delta x\right)=\Phi\left(\frac{\Delta z}{\Delta x^{H}}\right) .
$$


Simple algebra based on this scaling gives

$$
\sigma(\Delta x)=\ell^{1-H} \Delta x^{H},
$$

where $\sigma$ is the standard deviation of the height profile. Here $\ell$ denotes a length scale, also known as the topothesy. This quantity is defined by $\sigma(\ell)=\ell$, which allows the geometrical interpretation of the topothesy as the length scale over which the profile has a mean slope of 45 degrees. The smaller $\ell$, the flatter the profile appears on a macroscopic scale. In the case of a Gaussian height distribution, the probability $p(\Delta z ; \Delta x)$ reads:

$p(\Delta z ; \Delta x)=\frac{1}{\sqrt{2 \pi} \ell^{1-H} \Delta x^{H}} \exp \left[-\frac{1}{2}\left(\frac{\Delta z}{\ell^{1-H} \Delta x^{H}}\right)^{2}\right]$.

The self-affine profile is thus fully characterized by its exponent $H$, its topothesy parameter $\ell$ and the bounds of the self-affine regime $\xi_{-}$and $\xi_{+}$. Let us mention in addition that the above geometric interpretation of the roughness amplitude made above does not imply that $\ell$ has to lie between the lower $\left(\xi_{-}\right)$and upper $\left(\xi_{+}\right)$cutoff for the self-affine regime. For the surfaces usually considered in scattering problems, we rather expect that $\ell \ll \xi_{-}$. When $\xi_{-}<\ell<\xi_{+}$, the topothesy makes the transition between the scales, below $\ell$, for which a fractal dimension $D=2-H$ can be measured using the box counting method and the scales, above $\ell$, for which this dimension is just unity.

In the following we consider the scattering of spolarized electromagnetic waves from a one dimensional, random, self-affine surface. This surface, of Hurst exponent $H$, we denote by $z=\zeta(x)$, and it is assumed to be Gaussian self-affine. We will further assume that the lower limit of the self-affine regime is smaller than the wavelength, $\lambda$, of the incident wave. The scattering geometry considered is depicted in Fig. 11. The incident plane is assumed to be the $x z$-plane, and the rough surface, which is perfectly conducting, is illuminated from the vacuum side by a plane wave of frequency $\omega=2 \pi / \lambda$. The incident and scattering angle respectively we denote by $\theta_{0}$ and $\theta$, and they are defined positive according to the convention indicated in Fig. 1. In the above scattering geometry there is no depolarization, and the electromagnetic field is represented by the single non-vanishing component of the electric field $\Phi(x, z \mid \omega)=E_{y}(x, z \mid \omega)$, which should satisfy the (scalar) Helmholtz equation:

$$
\left(\partial_{x}^{2}+\partial_{z}^{2}+\frac{\omega^{2}}{c^{2}}\right) \Phi(x, z \mid \omega)=0,
$$

with vanishing boundary condition on $z=\zeta(x)$ and outgoing wave condition at infinity. In the far field region, above the surface, the field can be represented as the sum of an incident wave and scattered waves:

$\Phi(x, z \mid \omega)=\Phi_{0}(x, z \mid \omega)+\int_{-\infty}^{\infty} \frac{d q}{2 \pi} R(q \mid k) e^{i q x+i \alpha_{0}(q, \omega) z}$, where the plane incident wave is given by:

$$
\Phi_{0}(x, z \mid \omega)=\exp \left\{i k x-i \alpha_{0}(k, \omega) z\right\}
$$

and $R(q \mid k)$ is the scattering amplitude. In the above expression, we have defined $\alpha_{0}(q, \omega)=\sqrt{(\omega / c)^{2}-q^{2}}$ $\left(\Re \alpha_{0}(q, \omega)>0, \Im \alpha_{0}(q, \omega)>0\right)$. Furthermore, the momentum variables $q$ and $k$ are in the radiative region related to respectively the scattering and incident angle by $q=(\omega / c) \sin \theta$ and $k=(\omega / c) \sin \theta_{0}$, so that $\alpha_{0}(q, \omega)=(\omega / c) \cos \theta$ and $\alpha_{0}(k, \omega)=(\omega / c) \cos \theta_{0}$.

Our aim is to obtain an expression for the scattering amplitude, since this quantity determines the scattered field. The mean differential reflection coefficient (or mean scattering cross section), which is an experimentally accessible quantity, and defined as the fraction of the total, time-averaged, incident energy flux scattered into the angular interval $(\theta, \theta+d \theta)$, is related to this quantity by the following expression [26]:

$$
\left\langle\frac{\partial R}{\partial \theta}\right\rangle=\frac{1}{L} \frac{\omega}{2 \pi c} \frac{\cos ^{2} \theta}{\cos \theta_{0}}\left\langle|R(q \mid k)|^{2}\right\rangle .
$$

Here $L$ denotes the length of the self-affine profile function as measured along the $x$-direction, and the other quantities have been defined earlier. The angle brackets denote an ensemble average over the surface profiles $\zeta(x)$, and the momentum variables are understood to be related to the angles $\theta_{0}$ and $\theta$ according to the expressions given above.

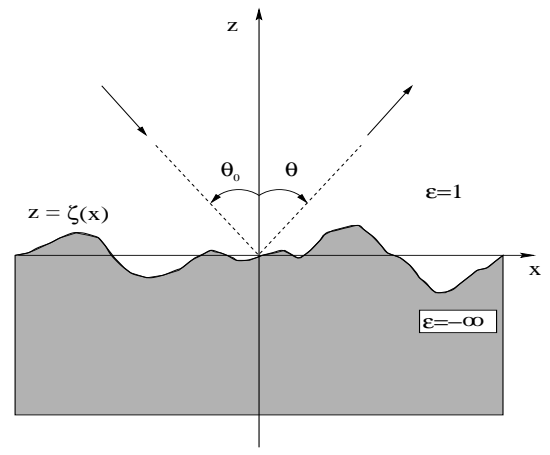

FIG. 1. Sketch of the scattering geometry.

We now use the Kirchhoff-approximation, which consists of locally replacing the surface by its tangential plane, and thereafter using the (local) Fresnel reflection coefficient for the local incident angle to obtain the scattered field. Notice here that dealing with a surface whose scaling invariance range is bounded by a lower cut-off $\xi_{-}$ does ensure that the tangential plane is well defined in every point. The scattering amplitude is then [26]:

$R(q \mid k)=\frac{-i}{2 \alpha_{0}(q, \omega)} \int_{-L / 2}^{L / 2} d x e^{-i q x-i \alpha_{0}(q, \omega) \zeta(x)} \mathcal{N}(x \mid \omega)$ 
where $\mathcal{N}(x \mid \omega)$ is a source function defined by $\mathcal{N}(x \mid \omega)=$ $\left.2 \partial_{n} \Phi_{0}(x, z \mid \omega)\right|_{z=\zeta(x)}$, with the (unnormalized) normal derivative given by $\partial_{n}=-\zeta^{\prime}(x) \partial_{x}+\partial_{z}$. By substituting this expression for the source function into Eq. (8), one can show after some straightforward algebra where one takes advantage of the self-affine profile function having stationary increments, that the mean differential reflection coefficient is given by

$$
\begin{aligned}
\left\langle\frac{\partial R}{\partial \theta}\right\rangle= & \frac{\omega}{2 \pi c} \frac{1}{\cos \theta_{0}} \frac{\cos ^{2} \frac{\theta+\theta_{0}}{2}}{\cos ^{2} \frac{\theta-\theta_{0}}{2}} \\
& \times \int_{-L / 2}^{L / 2} d v \exp \left\{i \frac{\omega}{c}\left(\sin \theta-\sin \theta_{0}\right) v\right\} \Omega(v),
\end{aligned}
$$

where

$$
\Omega(v)=\left\langle\exp \left\{-i \frac{\omega}{c}\left[\cos \theta+\cos \theta_{0}\right] \Delta \zeta(v)\right\}\right\rangle,
$$

with $\Delta \zeta(v)=\zeta(x)-\zeta(x+v)$. Note that the statistical properties of the profile function, $\zeta(x)$, enters Eq. (10) only through $\Omega(v)$. With Eq. (何), one may now calculate the ensemble average contained in $\Omega(v)$. For a Gaussian self-affine surface one gets:

$$
\begin{aligned}
\Omega(v) & =\int_{-\infty}^{\infty} d z \exp \left\{-i \frac{\omega}{c}\left(\cos \theta+\cos \theta_{0}\right) z\right\} p(z ; v) \\
& =\exp \left\{-\left(\frac{\omega}{c} \frac{\cos \theta+\cos \theta_{0}}{\sqrt{2}} \ell^{1-H} v^{H}\right)^{2}\right\}
\end{aligned}
$$

By performing the change of variable:

$$
u=v\left[\frac{\omega}{c} \frac{\cos \theta+\cos \theta_{0}}{\sqrt{2}} \ell^{1-H}\right]^{1 / H},
$$

in Eq. (10), and letting the length of the profile extent to infinity, $L \rightarrow \infty$, one finally obtains the following expression for the mean differential reflection coefficient:

$$
\left\langle\frac{\partial R}{\partial \theta}\right\rangle=\frac{a^{-\left(\frac{1}{H}-1\right)}}{\sqrt{2} \cos \theta_{0}} \frac{\cos \frac{\theta+\theta_{0}}{2}}{\cos ^{3} \frac{\theta-\theta_{0}}{2}} \mathcal{L}_{2 H}\left(\frac{\sqrt{2} \tan \frac{\theta-\theta_{0}}{2}}{a^{\frac{1}{H}-1}}\right),
$$

where $a=\sqrt{2} \frac{\omega}{c} \ell \cos \frac{\theta+\theta_{0}}{2} \cos \frac{\theta-\theta_{0}}{2}$, and $\mathcal{L}_{\alpha}(x)$ is the centered symmetrical Lévy stable distribution of exponent $\alpha$ defined as

$$
\mathcal{L}_{\alpha}(x)=\frac{1}{2 \pi} \int_{-\infty}^{\infty} d k e^{i k x} e^{-|k|^{\alpha}} .
$$

Note that in the above expressions the wavelength, $\lambda$, only comes into play through the combination $(\ell / \lambda)^{1-\frac{1}{H}}$ which appears both in the prefactor and in the argument of the Lévy distribution. This quantity can be geometrically regarded as $s(\lambda)^{1 / H}$ where $s(\lambda)=(\ell / \lambda)^{1-H}$ is the typical slope of the surface over a wavelength. The behavior of the scattered intensity is thus entirely determined by this typical slope $s(\lambda)$ and the roughness exponent H. Figure 2 shows the mean differential reflection coefficient (DRC), obtained for a incident angle of $\theta_{0}=50^{\circ}$ for different values of the ratio $\ell / \lambda$. Note that the Lévy distribution obtained above is likely not to be specific of the Kirchhoff approximation: the integrand in (10) is indeed nothing but the reflection of the statistical translational invariance of the problem.

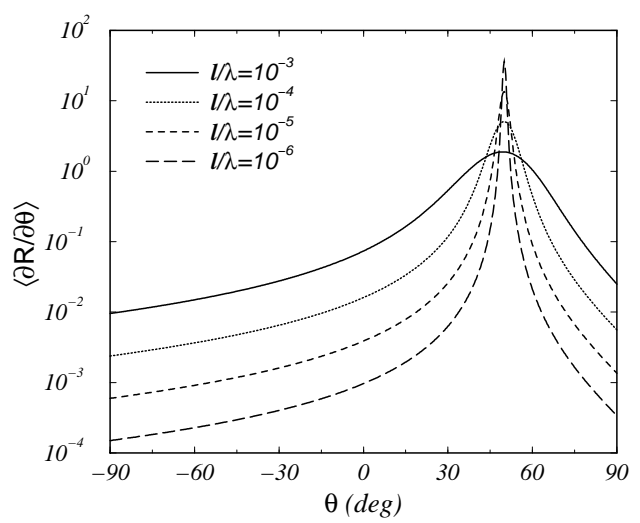

FIG. 2. The mean DRC as defined by Eq. (14) for an incidence angle of $\theta_{0}=50^{\circ}$ and a roughness exponent of $H=0.7$. The surfaces had topothesy over wavelength of $\ell / \lambda=10^{-3}$, $10^{-4}, 10^{-5}$ and $10^{-6}$ which gives typical slopes at the wavelength scale of respectively $s(\lambda)=0.126,0.063,0.032$ and 0.016 .

Taking advantage of the asymptotic expansion of the Lévy distribution around zero [27] we find that the amplitude of the specular peak scales as

$$
\left.\left\langle\frac{\partial R}{\partial \theta}\right\rangle\right|_{\theta=\theta_{0}} \simeq \frac{\Gamma\left(\frac{1}{2 H}\right)}{2 \sqrt{2} \pi H\left(2 \sqrt{2} \pi \frac{\ell}{\lambda} \cos \theta_{0}\right)^{\frac{1}{H}-1}},
$$

and its half width (assuming $\frac{\omega}{c} \ell \cos \theta_{0} \ll 1$ ) as

$$
w \simeq 2 \sqrt{\frac{\Gamma\left(\frac{1}{2 H}\right)}{\Gamma\left(\frac{3}{2 H}\right)}}\left(2 \sqrt{2} \pi \frac{\ell}{\lambda} \cos \theta_{0}\right)^{\frac{1}{H}-1} .
$$

Notice that in the case of a non zero angle of incidence, $\theta_{0}$, this (specular) peak is located at $\theta=\theta_{0}+\Delta \theta_{0}$, where $\Delta \theta_{0}\left(\Delta \theta_{0} \sim w^{2} \ll w\right)$ scales as

$$
\Delta \theta_{0} \simeq \frac{2 H-1}{H} \frac{\Gamma\left(\frac{1}{2 H}\right)}{\Gamma\left(\frac{3}{2 H}\right)} \tan \theta_{0}\left(2 \sqrt{2} \pi \frac{\ell}{\lambda} \cos \theta_{0}\right)^{\frac{2}{H}-2},
$$

i.e. we have, due to the self-affinity of the surface, a shift, $\Delta \theta_{0}$, of the specular direction as compared to its "classical" position $\theta=\theta_{0}$.

It is interesting that these non-trivial scaling results can all be retrieved via simple dimensional arguments. Let us examine the intensity scattered in direction $\theta$; in a naive Huyghens framework two different effects will compete to destroy the coherence of two source points on the surface $i$ ) the angular distance separating $\theta$ from 
the specular direction ii) the roughness. The two source points will interfere coherently if the distance between them is lower than the two length scales $d_{\text {ang }}=\lambda / p$ where $p=\tan \left[\left(\theta-\theta_{0}\right) / 2\right]$ is the slope of the isophase plane and $d_{\text {rough }}$, the typical length scale over which the height difference is of order $\lambda$. In case of a self-affine surface, $d_{\text {rough }}=\lambda^{\frac{1}{H}} \ell^{1-\frac{1}{H}}$. Depending on the observation angle $\theta$, the coherence will be controlled either by $d_{\text {rough }}$ or by $d_{a n g}$, which will correspond respectively to the peak or the tail of the intensity distribution. The width $w$ of the peak can then be defined as the transition between these two regions and will be such that $d_{\text {rough }} \simeq d_{\text {ang }}$. This leads directly to $w \propto(\ell / \lambda)^{\frac{1}{H}-1}$. While discussing the width of the peak present in the mean DRC, we also have to take into account the fact that real self-affine surfaces are not scale invariant over an infinite range of length scales. The upper cut-off $\xi_{+}$introduces a convolution of the preceding results by a function of width $\lambda / \xi_{+}$. This convolution does not affect the tails of the distribution but considering the peak we have now to distinguish two regimes whose transition will be defined by $\sigma=\ell^{1-H} \xi_{+}^{H} \simeq \lambda$ where $\sigma$ denotes here the standard deviation of the height profile measured over a macroscopic scale i.e. larger than $\xi_{+}$. For $\sigma<\lambda$ the width of the peak will be $w \simeq(\ell / \lambda)^{\frac{1}{H}-1} \simeq s(\lambda)^{\frac{1}{H}}$ and for $\sigma>\lambda$ we will get $w \simeq \lambda / \xi_{+}$.

Using now the expansion of the Lévy distribution at infinity 28] we get for the diffuse intensity $\left(\theta \neq \theta_{0}\right)$ :

$$
\left\langle\frac{\partial R}{\partial \theta}\right\rangle \simeq \frac{\Gamma(1+2 H) \sin (\pi H)}{4 \pi} \frac{\left(4 \pi \frac{\ell}{\lambda}\right)^{2-2 H}\left(\cos \frac{\theta+\theta_{0}}{2}\right)^{3-2 H}}{\cos \theta_{0}\left|\sin \frac{\theta-\theta_{0}}{2}\right|^{1+2 H}} .
$$

The dependence of the prefactor on the surface parameters $\ell$ and $H$ is a new result of this letter. If the scaling can be easily retrieved via a first order expansion in plane waves, the above expression suggests that provided single scattering is considered, the power law behavior survives even in a non perturbative approach.

We proposed a direct derivation of the intensity distribution of a wave scattered from a perfectly conducting self-affine rough surface in the framework of single scattering. Using a simple Kirchhoff approximation we obtain an exact solution consistent with all previously published perturbative or numerical results. Among other examples, our results could be directly tested in the case of an acoustic wave scattered from a fracture surface. This work will be extended to deal with more complicated problems directly related to scattering from rough surfaces (shadowing effects, multiple scattering, dielectric media...). Numerical investigations about the range of validity of the results obtained in this letter will follow.

The authors would like to thank Claude Boccara, Jean-Jacques Greffet, Tamara Leskova and Alexei A. Maradudin for useful comments about this work. I.S. would like to thank the Research Council of Norway
(Contract No. 32690/213), Norsk Hydro ASA, and Total Norge ASA for financial support.

[1] J.W.S. Rayleigh "The theory of sound", Dover, (NewYork, 1945).

[2] P. Beckmann and A. Spizzichino, "The scattering from electromagnetic waves from rough surfaces", Artech House, (1963).

[3] J. A. Ogilvy, Theory of wave scattering from random rough surfaces", IOP Pub., (Bristol, GB, 1991).

[4] M. Nieto-Vesperinas, "Scattering and diffraction in physical optics", Wiley, (1991).

[5] A.G. Voronovich, "Wave scattering from rough surfaces", Springer Verlag, (1994).

[6] B.B. Mandelbrot, "The fractal geometry of nature", Freeman (1975).

[7] B.B. Mandelbrot, D.E. Passoja and A.J. Paullay, Nature 308, 721, (1984).

[8] E. Bouchaud, E. Lapasset and J. Planés, Europhys. Lett. 13, 73, (1990).

[9] K.J. Måløy, A. Hansen, E.L. Hinrichsen and S. Roux, Phys. Rev. Lett. 68, 213, (1992).

[10] P. Meakin, "Fractals, scaling and growth far from equilibrium", Cambridge University Press, (1998).

[11] F. Plouraboué and M. Boehm, Trib. Int. 32, 45, (1999).

[12] D. Abriou, Y. Levillain, H. Arribart and F. Creuzet, Verre 2, 3, (1996).

[13] J. Schmittbuhl, Ph.D. thesis, Université Paris 6, (1994).

[14] M. V. Berry, J. Phys. A 12, 781-797, (1979).

[15] D.L. Jaggard and X. Sun, J. Appl. Phys. 68, 11, 5456, (1990).

[16] M.K. Shepard, R.A. Brackett and R.E. Arvidson, J. Geophys. Res. 100, E6, 11709, (1995).

[17] P.E. McSharry, P.J. Cullen and D. Moroney, J. Appl. Phys. 78, 12, 6940, (1995).

[18] N. Lin, H.P. Lee, S.P. Lim and K.S. Lee, J. Mod. Optics 42, 225, (1995).

[19] J. Chen, T.K.Y. Lo, H. Leung and J. Litva, IEEE Trans. Geosci. 34, 4, 966, (1996).

[20] C.J.R. Sheppard, Opt. Comm. 122, 178, (1996).

[21] J. A. Sánchez-Gil and J. V. García-Ramos, Waves Random Media 7, 285-293, (1997).

[22] J. A. Sánchez-Gil and J. V. García-Ramos, J. Chem. Phys. 108, 1,317, (1998).

[23] Y-P Zhao, C.F. Cheng, G.C. Wang and T.M. Lu, Surface Science 409, L703, (1998).

[24] E. Jakeman, in "Fractals in physics", L. Pietronero and E. Tossati Ed., Elsevier, (1986).

[25] D.L. Jordan, R.C. Hollins, E. Jakeman and A. Prewett, Surface topography 1, (1988).

[26] A.A. Maradudin, T. Michel, A.R. McGurn, and E.R. Mendez, Ann. Phys. 203, 255, (1990).

[27] At zero, $\mathcal{L}_{\alpha}(x)=\frac{1}{\pi \alpha} \Gamma\left(\frac{1}{\alpha}\right)\left[1-\frac{\Gamma\left(\frac{3}{\alpha}\right)}{2 \Gamma\left(\frac{1}{\alpha}\right)} x^{2}\right]+\mathcal{O}\left(x^{4}\right)$.

[28] At infinity $\mathcal{L}_{\alpha}(x)=\frac{\Gamma(1+\alpha)}{\pi|x|^{1+\alpha}} \sin \left(\frac{\alpha \pi}{2}\right)+\mathcal{O}\left(\frac{1}{|x|^{1+2 \alpha}}\right)$. 